\title{
APORTES PARA PENSAR LA CÁTEDRA DE LA PAZ DESDE LAS RESISTENCIAS Y PROYECTOS DE PAZ DE LOS GRUPOS ÉTNICOS EN COLOMBIA*
}

\author{
CONTRIBUTIONS TO THINK ABOUT THE TEACHING \\ OF PEACE FROM THE RESISTANCES AND PEACE \\ PROJECTS OF THE ETHNIC GROUPS IN COLOMBIA
}

\author{
MARÍA ISABEL VILLADA** \\ JUAN CAMILO ESTRADA***
}

Recibido: 20 de agosto de 2017 - Aceptado: 20 de septiembre de 2017 - Publicado 31 julio de 2018 DOI: $10.24142 /$ raju.v13n26a2

\section{Resumen}

Las reflexiones que presentamos en esta ponencia devienen de las indagaciones realizadas como parte de los proyectos de investigación que presentamos para optar al título de Licenciatura en Educación Básica con énfasis en Ciencias Sociales de la Universidad de Antio-

* Ponencia de reflexión teórica.

** Estudiante de Maestría en Historia y Memoria, Universidad Nacional de La Plata. Licenciada en Educación Básica con énfasis en Ciencias Sociales, Universidad de Antioquia. Facilitadora del proyecto Paz: aporte hacia la reconciliación de la Corporación Educativa Combos.

Correo electrónico: isabelvillada60@gmail.com

*** Estudiante de Maestría en Educación y Derechos Humanos, Universidad Autónoma Latinoamericana. Licenciado en Educación Básica con énfasis en Ciencias Sociales, Universidad de Antioquia. Docente e investigador, Facultad de Educación, Universidad de Antioquia.

Correo electrónico: juanc.estrada@udea.edu.co 
quia. A partir de la pregunta por el vínculo entre construcción de paz y las realidades interculturales presentes en los escenarios educativos, retomamos tres experiencias de construcción de paz y resistencia desde las comunidades étnicamente diferenciadas de nuestro país para analizar las posibilidades que presentan para transformar relaciones y contextos desde su inclusión en la configuración de la llamada "Cátedra de la paz".

Palabras clave: Proceso de paz en Colombia, comunidades indígenas, comunidades afrocolombianas, pueblo Rom, Cátedra de la paz.

\section{Abstract}

The reflections that we show in this presentation come from the investigations made as part of the research projects that we presented to obtain the bachelor's degree in basic education with emphasis in social sciences from the University of Antioquia. Based on the question about the link between peacebuilding and the intercultural realities that are present in the educational scenarios, we retake on 3 experiences of building peace and resistance from the ethnically differentiated communities of our country to analyze the possibilities they present to transform relationships and contexts since its inclusion in the configuration of the so-called "Teaching of peace".

Keywords: Peace process in Colombia; Indigenous communities; Afro-Colombian communities; Gypsy People; Teaching of peace 
Aportes para pensar la Cátedra de la paz desde las resistencias y proyectos de paz de los grupos étnicos en Colombia

\section{INTRODUCCIÓN}

En el proceso de paz que se llevó a cabo entre el grupo guerrillero de las FARC-EP y el gobierno de Colombia, denominado "Acuerdo General para la terminación del conflicto y la construcción de una paz estable y duradera", luego de seis meses de negociación en Oslo, Noruega, se dio a conocer al país el acuerdo general, iniciando el 19 de noviembre del 2012 en La Habana y finalizando el 26 de septiembre de 2016, con la firma protocolaria del Acuerdo Final de Paz entre el gobierno colombiano y las FARC-EP, en el Patio de Banderas del Centro de Convenciones Julio César Turbay, en Cartagena. De esta manera, el país se preparó para avalar, o no, por medio de un plebiscito refrendatario, los puntos de la agenda de negociación, a saber: desarrollo agrario integral, participación política, fin del conflicto, solución al problema de drogas ilícitas, víctimas, e implementación, verificación y refrendación. Este proceso de negociación se llevó a cabo con la ayuda de Noruega y Cuba, como países garantes, y el acompañamiento de los gobiernos de Venezuela y Chile. Es necesario saber que hasta el momento han sido expedidos treinta decretos que hacen parte de la implementación del acuerdo, entre los cuales Arenas (2017) afirma que:

Fue aprobada una ley estatutaria y hay otra en trámite (reforma política) así como dos leyes ordinarias; otras dos están en trámite. La ley estatutaria aprobada corresponde al estatuto de la oposición... la ley de amnistía hace parte de las leyes aprobadas, así como aquella que permite desempeñar su rol en el congreso a Voces de Paz (p. 2).

Si vemos el escenario completo, el balance arroja 38 normas aprobadas entre reformas constitucionales, reformas legales y leyes estatutarias. Además, se encuentran siete reformas en trámite en el Congreso, junto a otras quince que pasarán por este recinto con el debido fast track ${ }^{1}$ (vía rápida) que el gobierno prorrogó para llevar un proceso adecuado.

En este proceso, ha representado un hito en los avances para la paz que se haya considerado la participación, por primera vez, de las víctimas de la guerrilla de las FARC-EP, lo que deja entreabiertas las múltiples oportunidades para visibilizar las luchas y resistencias llevadas a cabo por las

1 Mecanismo contemplado en el acto legislativo número 1 de 2016, con el cual se busca reducir el número de debates en el Congreso. 
comunidades campesinas, ya que ha sido en las áreas rurales donde se han concentrado con mayor fuerza los enfrentamientos, pero también de aquellos pueblos que han sido étnicamente reconocidos por la Carta Constitucional de 1991, quienes desde sus territorios ancestrales, cosmogonías, saberes, prácticas y costumbres han construido su propia paz, considerando la polisemia del concepto en la medida en que, si la paz se entiende como un estado de cosas, como un camino en permanente construcción que nos posibilitará otra forma de vivir y convivir — no siendo entonces un fin o estadio último-, no se vive de igual manera en toda la población. Es por lo tanto menester que esta reflexión vincule los procesos educativos que, en el marco del Acuerdo de paz y en un posible escenario de posconflicto, ${ }^{2}$ se van a desarrollar por medio de la "Cátedra de la paz", que servirá, al mismo tiempo, como mecanismo para garantizar la no repetición de los hechos acontecidos durante el conflicto armado.

Así, pretendemos presentar, en primer momento, un breve marco conceptual que nos permita un entendimiento de la paz desde un enfoque diferencial y que se configure en las resistencias cotidianas que llevan a cabo las comunidades en sus territorios, al igual que reflexionar sobre las concepciones de justicia legal y justicia transicional que aporte al camino de la reconciliación, comprendiendo nuestro contexto. Posteriormente, exponemos las experiencias de paz, teniendo como referente los procesos de lucha por el buen vivir, haciendo alusión a los planes de vida que tienen los pueblos indígenas, la delimitación de territorios de paz que han sido así nombrados por las comunidades afrocolombianas y la Kriss Romaní, el mecanismo para la solución de conflictos al interior de las Kumpanías del pueblo rom (gitano). Por último, aportamos algunas consideraciones con respecto a la implementación de la Cátedra de la paz que se desprenden del conocimiento y reconocimiento de esas otras experiencias de construcción de paz, desde las realidades de los grupos étnicos, a partir de la idea de justicia epistémica y en consonancia con el logro de un empoderamiento pacifista.

2 Desde inicios del proceso de negociación, este concepto se ha puesto bajo sospecha debido al sentido de total ausencia de conflicto que en él subyace, por lo tanto, se ha propuesto también hablar de "Post-diálogos" o "Post-acuerdo", con los cuales se deja de manifiesto que la terminación del enfrentamiento armado no implica la ausencia total de conflicto, ya que este puede transitar a otros ámbitos; y considera también la existencia de otros actores armados. 
Aportes para pensar la Cátedra de la paz desde las resistencias y proyectos de paz de los grupos étnicos en Colombia

\section{RESISTENCIA(S), JUSTICIA Y PAZ DESDE UN ENFOQUE DIFERENCIAL}

Ante el escenario del posacuerdo, resultado de los diálogos de paz entre el gobierno colombiano y el grupo guerrillero de las FARC-EP, que busca abonar al camino para la construcción de una paz estable y duradera, cabe preguntarnos ¿qué es la paz? y ¿qué entendemos por paz? teniendo presente los lugares de enunciación que asumimos. En este sentido, la paz no puede entenderse simplemente como la ausencia de violencia, esto es, la paz como opuesta a la guerra. Es en todo caso un concepto sobre el cual no hay consenso pero sí múltiples interpretaciones. Retomamos una visión ampliamente reconocida y propuesta por Galtung (2003) para quien la paz representa la capacidad para manejar los conflictos con empatía, creatividad y sin recurrir a la violencia; esto implica sentir y entender las pasiones del otro sin tener que estar totalmente de acuerdo, además, superar las visiones polarizadas de las partes en conflicto para buscar nuevas alternativas. El mismo autor entiende la paz y la violencia en analogía con la salud y la enfermedad, siendo entonces estadios dialécticos; pero lo que nos interesa resaltar de esta concepción es que la violencia no se concibe simplemente como enfrentamiento armado, ya que la violencia puede manifestarse de múltiples formas sin tener que desembocar, necesariamente, en un enfrentamiento físico por medio de las armas.

Nuestra Carta Constitucional es llamada "la constitución para la paz", la cual, en su artículo 22, define que "la paz es un derecho y un deber de obligatorio cumplimiento" ya que se consagra en la construcción cultural, en la idea de un mundo nuevo, con el respeto de los derechos humanos como base, la solidaridad, la democracia, la amistad entre pueblos y naciones; por ello, se pretende asumir la paz como paradigma y distanciarse de la guerra y la violencia, para transformar los conflictos de manera creativa y lograr así la refundación de la nación a partir de una cultura para la paz. En este sentido, el Museo Casa de la Memoria de la ciudad de Medellín (2015) resalta que:

La paz es mucho más que el silencio del fuego. No es un acuerdo absoluto y unánime, ni un lugar al que se llega, en el que las personas son espectadores y no actores. Tiene una perspectiva utópica, y en cuanto tal plantea un horizonte, más que un punto de partida, pero se ve materializada en acciones concretas y cotidianas que le dan sentido real (p. 1). 
La paz, por lo tanto, no puede existir sin el respeto y total reconocimiento de los derechos humanos y, por ende, de la verdad, la libertad, la solidaridad, la seguridad y la justicia; sin embargo, teniendo en cuenta estas características, no es posible entender la paz de forma unívoca, ni pretender que ella sea asumida de la misma forma por diferentes personas, grupos sociales y comunidades que, aunque compartan elementos comunes como la nacionalidad y el idioma, sus construcciones simbólicas y visiones de mundo son estructuralmente distintas. Por ello abogamos por una mirada a la paz desde un enfoque diferencial.

El enfoque diferencial, en el marco del proceso de paz, parte de la singularidad de los daños, "nace con la pretensión de ofrecer un panorama de restitución de derechos a víctimas del conflicto desde sus particularidades; parte, por tanto, de la noción de diversidad y vulnerabilidad de estas personas" (Arteaga, 2012, p. 15). Este enfoque surge como respuesta a las condiciones multiculturales del país que fueron constitucionalmente reconocidas en 1991, lo que lleva, inherentemente, a considerar la autonomía y derechos diferenciados de los grupos étnicos, especialmente en lo concerniente al territorio, ya que este es un elemento central para la vida y cosmogonía de dichas comunidades. Por lo tanto, la paz y la forma como se vive y se asume va a depender radicalmente de las formas propias de existencia de cada grupo, como aquellos que han sido excluidos del marco estatal producto de un racismo estructural. Siguiendo a Arteaga (2012):

Se puede afirmar que la relación entre enfoque diferencial y construcción de paz está dada por una doble condición: de un lado, implica que difícilmente puede alcanzarse la paz sin que se adopten posiciones políticas acordes con la satisfacción de las necesidades de cada uno de los grupos diferenciados, en atención a sus particularidades étnicas, culturales, sociales y de género, entre otras. De otra parte, implica que una adecuada y efectiva implementación del enfoque diferencial favorecerá que se evidencien más oportunidades de alcanzar la paz en los territorios (p. 34).

Esta idea de paz con enfoque diferencial está ligada fuertemente a los procesos históricos de resistencia por parte de los grupos étnicos en Colombia, quienes, además de enfrentar a los actores armados partícipes del conflicto (guerrillas, paramilitares, ejército), han tenido que hacer frente a los embates de un sistema económico que incursiona en sus territorios, violentando sus formas de organización, prácticas culturales y productivas. 
En el caso de las comunidades afrocolombianas afirma Escobar (2014), que "para el año 2000, la mayoría de los territorios colectivos de afrodescendientes en el Pacífico había pasado a ser parte del escenario de guerra" (p. 71). Esta realidad, que se puede hacer extensible a diversos territorios habitados por comunidades indígenas y afrodescendientes, ha generado la necesidad de configurar proyectos propios, declarando que la guerra que día a día deja más víctimas no les corresponde y que, por ende, se distancian de todo actor armado porque va en contra de sus proyectos de vida. Retomando a Porto-Goncalves y Leff, Escobar (2012) argumenta que "estos grupos no solamente resisten el despojo y la des-territorialización, ellos redefinen sus formas de existencia a través de movimientos emancipatorios y la reinvención de sus identidades, sus modos de pensar, y sus modos de producción y de sustento" (p. 93).

Entonces, resulta imprescindible observar cómo la paz y la justicia se anudan para llevar a cabo un proceso de reconciliación. No obstante, es necesario dar un vistazo a las concepciones que circulan en nuestro contexto frente a la justicia, y es que, si bien el Estado Social de Derecho construye la justicia desde un cúmulo de leyes, esta no ha sido la misma ni la única forma en la que las comunidades visibilizan y ejecutan la justicia en sus territorios.

La justicia legal se diferencia de la justicia transicional en tanto la primera se vale de "las leyes para lograr la justicia, es el castigo, por ello aquí se habla de una justicia fundamentalmente punitiva. Así se dice que si no hay justicia se está entronizando la impunidad" (Restrepo, 2013, p. 1). Además de estar centrada en el victimario, donde este paga una pena por su falta, no hay interés en el arrepentimiento, perdón y reincorporación desde y hacia la sociedad. La segunda, se fundamenta en la justicia moral, es necesaria la visibilización de las víctimas donde se dotan de reconocimiento e identidad, se buscan las acciones de no repetición, dando relevancia al perdón, al respeto y valoración que generan las condiciones para que estos hechos no sucedan nuevamente. La importancia en la justicia transicional refiere a los procesos de verdad, donde:

El castigo no recae sobre el crimen sino sobre quién lo perpetró; igualmente, el perdón no recae sobre el crimen que es imperdonable, sino sobre el agente. Así el poder del perdón permite desligar al agente de su acto, para dar lugar a su reconocimiento, al de su dignidad: tú eres mejor que tus acciones (Ricoeur, 2000 citado en Restrepo, 2013, p. 6). 
El compromiso con la verdad, la reparación y la no repetición, son los elementos base y marcan el camino de la justicia transicional hacia la reconciliación, teniendo en cuenta las diferentes construcciones morales de las comunidades étnicamente diferenciadas en sus territorios. Es así que Restrepo (2013) afirma que "resultan más efectivos el perdón y la reconciliación que abren posibilidades de una nueva vida, que el castigo que retrotrae siempre al delito cometido" (p. 7).

En esta medida es necesario reflexionar sobre los hechos victimizantes de nuestro conflicto armado, y las particularidades que los habitantes de diferentes grupos étnicos han encontrado para realizar acciones de perdón, de justicia y resistencia buscando la paz, permitiendo observar que la justicia legal, con sus bloques legislativos, no accede a observar las particularidades de las cosmogonías frente a la justicia y paz, no repara ni reconoce las condiciones de no repetición necesaria para el conflicto armado como el colombiano. Es muy importante tener presente que este proceso de negociación, llevado a cabo entre el gobierno y las FARC-EP, ha implicado un reconocimiento especial de las víctimas y, en esa medida, debemos observar otras formas de alteridad y reivindicación de la dignidad como paso hacia la reconciliación.

\section{TRES EXPERIENCIAS DE CONSTRUCCIÓN DE PAZ: PLANES DE VIDA, TERRITORIOS DE PAZ Y SOLUCIÓN DE CONFLICTOS}

"Los pueblos indígenas hemos construido y vivido la paz todo el tiempo", expresaba Aida Quilcue, lideresa del pueblo nasa y coordinadora del programa de paz del Consejo Regional Indígena del Cauca (CRIC) durante el lanzamiento de la propuesta de paz del Movimiento Indígena el 14 de diciembre de 2012, en territorio ancestral del resguardo La María en Piendamó, Cauca; evento en el que participaron, además de los representantes de diversas organizaciones indígenas de todo el país, el Programa de las Naciones Unidas para el Desarrollo (PNUD), Indepaz, el Consejo de Paz Noruego, la Ruta Pacífica de las Mujeres e, incluso, el exmilitante del M-19 Antonio Navarro, quien resaltó el papel de la Guardia Indígena como actor de paz ("Propuesta de paz del Movimiento Indígena, una iniciativa para todos", 2012). Este evento se llevó a cabo apenas dos meses después de iniciados los diálogos de paz entre el gobierno colombiano y las FARC-EP en La Habana, con el cual se pudo demostrar una vez más la existencia de procesos 
de construcción de paz desde las comunidades (no solo los pueblos indígenas, sino también las comunidades afrocolombianas y campesinas), quienes han trabajado en la consecución de un buen vivir pero, al mismo tiempo, han luchado y resistido los embates de diferentes actores que incursionan en sus territorios motivados por intereses políticos y económicos.

Los pueblos indígenas, el pueblo rom y las comunidades afrodescendientes en el territorio colombiano, a lo largo de la historia, han sido víctimas de violencias de diversos tipos ligadas a las condiciones de la época: esclavitud durante la colonia, malas condiciones de vida, racismo, discriminación y exclusión durante los siglos XIX y XX. Hoy en día, cuando el país yace bajo el velo de un conflicto armado, son estas comunidades y pueblos, normalmente asentados en el campo, víctimas potenciales de la guerra, en la cual todas las partes combatientes comparten la barbaridad en sus acciones. Sin embargo, además de enfrentar las consecuencias de la guerra y las arremetidas de violencia en los territorios, otras formas de "no vivir en paz" permanecen.

En varias subregiones de Chocó y el Urabá miles de mujeres y hombres continúan hoy en día defendiendo sus vidas, territorios y culturas a través de proyectos alternativos de uso y manejo de recursos naturales, creando "zonas humanitarias", "comunidades de paz" y "zonas de biodiversidad" como alternativa a la devastación causada por el desarrollo promovido por actores vinculados con los mercados globales (Escobar, 2014, p. 72).

En este contexto, los planes de vida surgen como posibilidad para pensar la vida en torno a la organización comunitaria y el aprovechamiento colectivo de los frutos de la madre tierra. Bolaños y Pancho (2008) afirman que:

El desarrollo de los planes de vida ha implicado redefinir la proyección de los recursos financieros considerando propuestas globales que generen impactos más significativos en el bienestar integral de las comunidades [...] Sin embargo, es de anotar que esta perspectiva de los planes de vida es aún muy incipiente ante la prevalencia del modelo homogeneizador que sigue impactando en los territorios indígenas (p. 50).

Los planes de vida de las comunidades indígenas se construyen en un proceso participativo; es, por lo tanto, un acuerdo social que surge del con- 
senso para determinar cuál será el devenir de la comunidad. Un instrumento político que comprende información sobre los recursos y necesidades de la comunidad, así como los cambios que se esperan lograr y los mecanismos para ello, generando una posición con respecto a la relación entre el gobierno y otras instituciones que tengan cierto tipo de intervención en el territorio.

Los planes de vida surgen de las necesidades de las comunidades indígenas en relación con las políticas estatales. Rosero y Sánchez (2009) afirman que, hasta antes de la Constitución de 1991 los pueblos indígenas no aparecían en los planes de desarrollo de los departamentos, intendencias y municipios:

Los indígenas comienzan a hablar de planes de vida luego de que el gobierno de César Gaviria sanciona la ley 152 de 1994: Ley Orgánica de Plan de Desarrollo [...] A través de la ley, el Estado define que los pueblos indígenas deben formular sus propias políticas. En el X Congreso del CRIC en 1997 se recomendó a los cabildos indígenas utilizar el término "Plan de Vida" en vez de utilizar "Plan de Desarrollo": "Para que podamos construir nuestro destino desde nuestra propia lógica" (p. 7).

La Organización Nacional Indígena de Colombia (ONIC) plantea que los planes de vida pretenden "preservar la integridad étnica y cultural de los pueblos diseñada por ellos mismos con la dirección de sus autoridades, con el propósito de crear las condiciones para su desenvolvimiento futuro como grupos sociales y culturales distintos" (Rosero y Sánchez, 2009, p. 8).

La historia de los pueblos indígenas ha estado marcada por la lucha para sobrevivir y permanecer en el tiempo, conservando sus prácticas ancestrales, la lengua y sus saberes. En la actualidad las condiciones han cambiado poco; las políticas públicas, las leyes y la intervención estatal en los territorios autónomos han generado la necesidad de desplegar mecanismos políticos como el plan de vida, para sortear las necesidades y problemáticas de la comunidad y la región en relación con las imposiciones gubernamentales. "En los últimos años las comunidades han tenido que cambiar sus formas de vivir y de organizarse para poder preservar su vida en medio del conflicto social y armado, y el modelo de desarrollo" (Barrios y Hoyos, 2008, p. 96).

El plan de vida se concibe, entonces, como una herramienta integral de las diversas dimensiones que componen la vida de los pueblos: la cosmovisión, la historia y el diálogo intercultural con las personas no indígenas, 
Aportes para pensar la Cátedra de la paz desde las resistencias y proyectos de paz de los grupos étnicos en Colombia

lo que debe llevar a un buen vivir, en el que "reconozcamos que vivimos en un mundo de simbiosis, en donde cada quien es incompleto y requiere de "lo otro' para complementarse" (Giraldo, 2014, s. p.).

Una segunda propuesta, la iniciativa por declarar un territorio como "comunidad de paz", obedece a la intención mancomunada de las personas que lo habitan, quienes sufren de manera directa el impacto de la violencia. En Colombia no existe un consenso en torno al concepto ni a la forma de crear comunidades de paz; sin embargo, Hernández (2000) propone una definición que abarca características de algunos casos específicos:

\begin{abstract}
Iniciativas de paz desde la base, en construcción, que encuentran su origen en regiones de alta violencia y en comunidades que en medio del fuego cruzado se organizan como expresión de resistencia civil no violenta al conflicto interno armado, dentro de un territorio delimitado que declaran al margen de las hostilidades, para auto-protegerse reivindicando la autonomía e inmunidad de la población civil frente al conflicto armado, reconstruir el tejido social, desarrollar un proyecto de vida soportado en la solidaridad y el bien común, y materializar su derecho a la paz (p. 44).
\end{abstract}

Estas comunidades manifiestan su posición política no violenta hacia todos los actores del conflicto, tanto los grupos insurgentes y paramilitares como la fuerza pública, por acción u omisión de sus responsabilidades. La aplicación de estas estrategias de resistencia en el marco de la construcción de paz va acompañada por tácticas de seguridad alimentaria, que garantizan la permanencia en el territorio. Esto ha implicado recuperar semillas tradicionales, rescatar antiguos cultivos y buscar nuevas fuentes de alimentación: "el caso paradigmático es el de las comunidades negras del río Yurumanguí que después de treinta años, ante el cerco alimentario a que han estado sometidas por la guerra, han vuelto a sembrar arroz usando las semillas tradicionales" (Castillo, 2007, p. 158).

El aumento de las concesiones mineras, la fumigación como estrategia para la erradicación del cultivo ilícito de coca y amapolares, la militarización de los territorios y los macroproyectos de desarrollo, son factores que han potenciado la vulneración de derechos humanos y al territorio de las comunidades afrocolombianas en el presente siglo, como lo expresa Escobar (2014).

Ahora bien, una tercera experiencia nos acerca a la historia del pueblo Rom que, por su naturaleza ágrafa e itinerante, ha hecho difícil la labor de 
reconstruir el devenir de este mítico pueblo desde su salida del norte de la India, hace unos mil años; momento en el cual comenzó su dispersión por todo el mundo occidental. La vida de las personas rom en Colombia ha estado marcada por la exclusión y una suerte de racismo que ha violentado su cotidianidad, ya que la tradición ha enseñado que son ladrones, brujos, mentirosos, etc., lo que ha llevado a que opten por invisibilizarse y, prácticamente, romper toda relación no estrictamente necesaria con los gadye (no rom), así como no adherirse al sistema educativo nacional, que consideran nocivo para las tradiciones y prácticas propias de su cultura y, aún más importante, para su lengua, pues al no contar con escritura corre más riesgo de desaparecer. Muchas de las personas rom viven en condiciones poco dignas; la mayoría no tiene un grado alto de escolaridad y su filiación con el sistema de salud es escasa o nula (Gómez, 2010); sumado a eso, la visión que tiene la población mayoritaria sobre este pueblo es negativa, lo que les dificulta realizar las labores que históricamente han sido su sustento, como es el caso de la quiromancia o lectura de la mano.

No obstante, una práctica que ha permanecido, a pesar de las inclemencias de la historia del pueblo rom es la Kriss Romaní, siendo esta la forma propia de solucionar los conflictos que se presentan al interior de la kumpanía en la cual, el consejo de ancianos que portan la sabiduría y la tradición oral son los encargados de mediar para restaurar la armonía y el equilibrio del grupo (Gamboa y Rojas, 2005). Con la Kriss Romaní se busca resolver las disputas relacionadas con la familia o entre familias, pero esta no tiene un carácter punitivo sino reparativo y armonizador, que va de la mano con las costumbres del pueblo rom.

La Kriss Romaní ha sido un mecanismo de invisibilización y protección, una estrategia etnocéntrica de sobrevivencia que fortalece los lazos al interior de la comunidad y les permite desaparecer de la vista de quienes los han perseguido; no obstante, las transformaciones sociales que han tenido lugar al interior de las Kumpanías han llevado a que los rom quieran ser visibles de nuevo y hacer parte de la vida política del país, por lo tanto, se ha visto la necesidad de establecer el diálogo intercultural con los gadye. En los últimos años el Proceso Organizativo del Pueblo Rom (PROROM) ha encaminado acciones para mejorar y solucionar las problemáticas existentes al interior de las Kumpanías y que afectan la vida de las personas; de igual forma, han avanzado en el diálogo con los entes gubernamentales para mejorar sus condiciones laborales, educativas y de salud. 
Aportes para pensar la Cátedra de la paz desde las resistencias y proyectos de paz de los grupos étnicos en Colombia

Volver sobre estas experiencias nos permite considerar los procesos de construcción de paz que cada comunidad y pueblo lleva a cabo a partir de sus particularidades y necesidades, acudiendo a mecanismos propios de su tradición y prácticas ancestrales para responder a problemas locales ligados a las realidades globales, que deben ser analizados desde una perspectiva negociada de autoridades locales/tradicionales y autoridades formales en el marco del aparato estatal.

\section{APORTES PARA PENSAR LA CÁTEDRA DE LA PAZ}

Colombia es un país con abundante normatividad para la atención integral a la población víctima del conflicto armado en sus diferentes manifestaciones. En el escenario actual, se busca generar transformaciones a nivel constitucional a través de la justicia transicional, la atención a los victimarios por medio de la inserción social, liderada por la Asociación Colombiana para la Reintegración (ley 1424 de 2010), las iniciativas para la reparación de 125 municipios por parte de la ONU; ${ }^{3}$ y en materia de educación, con la implementación de la Cátedra de la paz. A pesar de tales esfuerzos, existen grandes paradojas y permanecen las incertidumbres con respecto a la atención de la población diversa que habita nuestro territorio. Por esta razón, es necesario cuestionar algunas de las propuestas del gobierno actual, como promulgar la ley 1732 de 2014 y su posterior reglamentación con el decreto 1038 de 2015, que establece la obligatoriedad de implementar la Cátedra de la paz en todas las instituciones de educación primaria, secundaria y media del país, con el propósito de emprender la configuración de una cultura de la paz desde el sector educativo, permitiendo nuevos escenarios para las generaciones más jóvenes y las garantías de no repetición de los hechos acontecidos en el devenir del conflicto.

En el decreto 1038 de 2015, a través del cual se reglamenta la implementación de la Cátedra de la paz, se proponen tres aspectos fundamentales a profundizar. En primer lugar, se presentan los componentes que hacen parte del corpus de conocimiento que deben ser vinculados en las instituciones educativas del país a través de la cátedra, estos son: Cultura de la paz, entendida como el sentido y vivencia de los valores ciudadanos, los derechos

3 Son 125 de 1.122 municipios del país. Se trata de las poblaciones donde, según distintas agencias del Sistema de las Naciones Unidas, se concentrará el posconflicto en Colombia ("Estos son los 125 municipios del posconflicto, según la ONU”, 2015). 
humanos, el Derecho Internacional Humanitario, la participación democrática, la prevención de la violencia y la resolución pacífica de los conflictos, también se considera la Educación para la paz, que implica la apropiación de conocimientos y competencias ciudadanas para la convivencia pacífica, la participación democrática, la construcción de equidad, el respeto por la pluralidad, los derechos humanos y el Derecho Internacional Humanitario y, por último, el Desarrollo sostenible, siendo aquel que busca el crecimiento económico, la elevación de la calidad de la vida y el bienestar social, sin agotar la base de recursos naturales renovables en que se sustenta, ni deteriorar el ambiente o el derecho de las generaciones futuras a utilizarlo para la satisfacción de sus propias necesidades, de acuerdo con el artículo 3 de la ley 99 de 1993.

En segundo lugar, se busca transversalizar lo anterior con áreas del conocimiento escolar, tales como: ciencias sociales, historia, geografía, constitución política y democracia; ciencias naturales y educación ambiental o educación ética y en valores humanos. Finalmente, para alcanzar este propósito, las entidades territoriales certificadas en educación, en trabajo articulado con los Comités Territoriales de Capacitación a Docentes y Directivos Docentes, deben tener en cuenta la formación de docentes y directivos docentes en servicio adscritos a la entidad territorial en materia de derechos humanos, cultura de paz y competencias ciudadanas para la convivencia pacífica, la participación democrática, la diversidad y pluralidad; además, deben financiar o diseñar, en sus respectivos planes de formación a docentes y directivos docentes, programas y proyectos de alta calidad que ofrezcan las Instituciones de Educación Superior (IES) y otros organismos, para responder a los objetivos de la Cátedra de la paz, así como promover su incorporación. La implementación de este programa de formación, según el decreto en mención, debe contemplar un proceso de valoración y evaluación cada dos años, mediante mecanismos adecuados y contextualizados para determinar el impacto de los programas y proyectos de formación a docentes y directivos docentes.

La revisión del decreto, que demarca el camino para la configuración e implementación de la Cátedra de la paz, permite evidenciar que la misma será producto del trabajo mancomunado entre instituciones educativas, sean ellas escuelas o universidades, organizaciones civiles y entes gubernamentales. La cátedra no es un objeto terminado, aún requiere ser dotada de contenido y contextualizada atendiendo a las necesidades particulares de los territorios. En el escenario del posacuerdo, resultado de las negociaciones y 
Aportes para pensar la Cátedra de la paz desde las resistencias y proyectos de paz de los grupos étnicos en Colombia

la puesta en marcha de los puntos acordados en La Habana, aparece la educación como un reto, un medio y a la vez una necesidad para construir una paz estable y duradera; por ello, Urrego y Marín (2013) proponen una serie de aspectos clave a tener en cuenta para tal labor: formar sujetos críticos y reflexivos para participar activamente de la vida política del país, implementar proyectos educativos que apunten a la construcción de la memoria histórica, fortalecer el tejido social y comunitario a partir del vínculo escuela-sociedad, formar en competencias para tramitar los conflictos y deconstruir los imaginarios colectivos. Las autoras también confieren vital responsabilidad a la formación de maestros en las facultades de educación y, por supuesto, a los estamentos gubernamentales y administrativos.

En este momento crucial que atraviesa el país, diferentes posturas se encuentran frente a esta propuesta con respecto a su posible ejecución en las distintas aulas educativas. De estas reflexiones que le apuestan a la Cátedra de la paz encontramos algunas, como la del Centro Nacional de Memoria Histórica $(\mathrm{CNMH})$ con la caja de herramientas "Un viaje por la memoria histórica", con la cual se busca reconocer al sector educativo como un vehículo fundamental en la construcción de ciudadanía y consciente de las afectaciones del conflicto armado; la propuesta del Museo Casa de la Memoria de Medellín para la formación de docentes por medio de laboratorios pedagógicos y, también, las reflexiones por parte de maestros del sector oficial presentadas en el documento Orientaciones básicas para la implementación de la cátedra de la paz en las instituciones educativas de Medellín (García y Ospina, 2016); no dejando de lado las reflexiones que vinculan la preocupación existente por parte de los docentes ante el difuso escenario que plantea la cátedra, ya que al ser autónoma representa una carga laboral dentro de los quehaceres de la profesión. Sin embargo, la Cátedra de la paz se presenta como oportunidad para reflexionar sobre las relaciones sociales y favorecer una cultura de paz a partir de la cual se propone discutir las causas y consecuencias del conflicto armado, el cual tiene formas de existir instaladas en la sociedad que denotan efectos como la naturalización de la violencia, la deslegitimación del Estado, la descomposición del tejido social, la crisis de la democracia y, ante todo, una mirada de espaldas "al otro". Con respecto a este último aspecto, en lo que refiere a la alteridad, "la cátedra de la paz puede convertirse en un espacio para sacar del olvido las historias silenciadas" (García y Ospina, 2016, p. 2).

En este orden de ideas, la Cátedra de la paz no debe dejar de lado las concepciones de paz que poseen y viven las comunidades étnicas en sus 
territorios, y que en muchas ocasiones responden a la singularidad del daño que han decidido reparar de manera colectiva. Si bien, esta pretende brindar herramientas para cimentar una cultura de paz y fortalecer desde la escuela los procesos que se vienen dando en el país, es de vital importancia que sirva igualmente para reflexionar dichos asuntos y no encasillarla como otras cátedras en contenidos dentro de una malla curricular, o como un asunto discursivo contractual que esté guiado por premuras dentro del Acuerdo de paz. Es muy importante que la cátedra trascienda los muros de la escuela y se materialice en diferentes contextos.

La Cátedra de la paz debe ser un espacio de reflexión vivencial, se debe dinamizar esta iniciativa con los aprendizajes de quienes han liderado ejercicios en construcción de paz y que han acumulado múltiples experiencias al respecto. Esto lleva a considerar que la paz no comienza a construirse con el pacto que pone fin al enfrentamiento armado, o con la implementación de la cátedra en las instituciones educativas; por el contrario, la paz se ha ido construyendo de diferentes maneras, convocando grupos y pueblos en los territorios que afrontan múltiples formas de la violencia, como es el caso de los pueblos indígenas y afrodescendientes que resisten cotidianamente la incursión del frente estatal con sus políticas de desarrollo económico y que, por tal razón, se han propuesto configurar modelos de educación propia, una realidad que no puede ser marginal al introducirse la Cátedra de la paz. Por esta razón, apostamos a que la cátedra sirva como base para que los sujetos y comunidades -étnicamente diferenciadas o no-desarrollen un empoderamiento pacifista, el cual representa un "reconocimiento de las realidades, prácticas y acciones pacifistas y sus capacidades para actuar y transformar su entorno más o menos cercano" (Muñoz, Herrera, Molina y Sánchez, 2005, p. 138).

Este concepto da cuenta de "procesos perfectibles que permiten el desarrollo de capacidades y competencias individuales, comunitarias o colectivas para construir paces imperfectas o inacabadas; reconocen y posicionan experiencias de la misma naturaleza" (Hernández, 2015, p. 183) que, para el caso de Colombia, afirma la autora, se materializa en procesos de construcción de paz de pueblos indígenas y afrodescendientes, comunidades campesinas, organizaciones de víctimas y de mujeres.

Desde nuestra perspectiva, este proceso de construcción de la cátedra, con los propósitos que ya hemos expuesto, debe estar aunado a una idea de justicia cognitiva o epistémica desde la cual se reconozca que los saberes y apuestas de conocimiento de las comunidades son igualmente valiosos 
cuando se equiparan con aquellos conocimientos producidos desde la racionalidad científica occidental. En este sentido, apostamos por la idea de ecología de saberes que propone De Sousa (2009), la cual representa "la inagotable diversidad epistemológica del mundo" (p. 111).

El autor resalta que, en esta propuesta de ecología de saberes, la ignorancia es esencial ya que representa el potencial de aprendizaje y el reconocimiento de los saberes que circulan en la sociedad, así:

Lo que aprendemos es proporcional a lo que ignoramos, y lo ideal es que mantengamos la posibilidad de una constelación de saberes, de una configuración de saberes, porque el hecho de que lo que sabemos no pueda coexistir tiene consecuencias en la vida cotidiana (De Sousa, 2009, p. 124).

Reconocer estos saberes-otros que han estado ausentes en el proyecto de escolaridad, a través de la cátedra, posibilitará la configuración de un espacio de conocimiento intercultural y de diálogo de saberes, en el cual se dé valor a las manifestaciones epistémicas de los pueblos y comunidades que, a pesar de haber estado presentes en el desarrollo de la historia nacional, han estado ausentes en la construcción de la identidad nacional a través de la educación.

Para concretar la propuesta de ecología de saberes, De Sousa (2010) manifiesta la necesidad de contar con un proceso de traducción intercultural, "entendida como el procedimiento que permite crear inteligibilidad recíproca entre las experiencias del mundo, tanto las disponibles como las posibles" (pp. 45-46); por lo tanto, a ningún corpus de conocimiento se le otorga el estatuto de exclusividad, ni se considera completo totalmente, ya que

las experiencias del mundo son tratadas en momentos diferentes del trabajo de traducción como totalidades o partes y como realidades que no se agotan en esas totalidades o partes. Por ejemplo, ver lo subalterno tanto dentro como fuera de la relación de subalternidad (De Sousa, 2010, p. 46).

El propósito de llevar a cabo la traducción intercultural entre los saberes que se colocan en simultaneidad es "crear justicia cognitiva a partir de la imaginación epistemológica" (De Sousa, 2006, p. 105). Poner en diálogo tales perspectivas y sus actores implica abonar el camino para lograr una justicia global realmente democrática, en la cual se le dé voz autorizada a los 
sujetos que participan en tal proyecto, en este caso, hablando concretamente de la Cátedra de la paz, es menester que las comunidades étnicas participen en la construcción de la misma, aportando los saberes y prácticas que les han permitido vivir en paz dentro y fuera de los límites — reales y simbólicosde sus territorios.

En consecuencia, la cátedra no puede convertirse solo en la enseñanza de la historia del conflicto armado, exponiendo casos emblemáticos, no debe caer en la enseñanza de valores a partir de la reproducción enciclopédica de definiciones, en presentar formas de resolución de conflictos, o en un proyecto de convivencia escolar, teniendo en cuenta que cada aspecto mencionado anteriormente ya debe estar reemplazado por otras áreas del conocimiento dentro del proceso de enseñanza y aprendizaje en la escuela.

Si entendemos la multiplicidad de manifestaciones que tiene la paz y, al mismo tiempo las formas de no vivir en paz, la cátedra debe representar un elemento clave para el reconocimiento de la pluralidad de nuestro país, que vinculando las experiencias presentadas sea un vehículo para el respeto de los derechos humanos, comprender y ligar el acumulado de experiencias desde los territorios diversos, pero ante todo, la posibilidad de empoderar a las personas para que se arriesguen a ser partícipes en la construcción de un mundo nuevo para todos.

\section{CONFIGURACIÓN E IMPLEMENTACIÓN DE LA CÁTEDRA DE LA PAZ}

Para finalizar esta reflexión consideramos necesario identificar algunas pistas metodológicas para promover y vivir escenarios de convivencia y reconciliación en la experiencia cotidiana de la escuela, a partir de la transversalización de la Cátedra de la paz, y con el propósito de cimentar una cultura de paz a partir de la cual transformar, progresivamente, las percepciones y actitudes de la comunidad educativa, desnaturalizando algunas prácticas e instaurando otras que permitan caminar hacia espacios más solidarios y pacíficos. En este sentido, apostamos por dos vías de acción para que la cátedra sea una experiencia vivida en la escuela. En primer lugar, brindamos unas pistas metodológicas o estrategias para transversalizar las actitudes, prácticas y saberes propios de la cátedra en las diversas áreas del saber escolar; y, en segundo lugar, presentamos una propuesta para la construcción de un ambiente de aula pacífico, retomando elementos de las tres experiencias de paz desde los grupos étnicos. 
Aportes para pensar la Cátedra de la paz desde las resistencias y proyectos de paz de los grupos étnicos en Colombia

\section{PISTAS METODOLÓGICAS PARA TRANSVERSALIZAR LA CÁTEDRA EN EL CURRÍCULO}

Consideramos que esta apuesta para construir la paz desde la escuela y garantizar la no repetición de los hechos violentos no se reduce a una serie de contenidos o temas, ya que "la construcción de una cultura de paz no está centrada en nodos de información, sino en la estructuración de creencias, reacciones emocionales y respuestas verbales y comportamentales en procura del bienestar personal y social" (Peñas, 2015, s. p.); por lo tanto, proponemos un conjunto de estrategias que sean aplicables a todas las asignaturas que componen el plan de estudios, que puedan ser llevadas a cabo por docentes, personal directivo y estudiantes, para que la paz se convierta en un eje articulador del quehacer en la escuela. Estas son:

- Promover acciones comunicativas y formativas conjuntas para la divulgación de experiencias de educación para la paz en el marco de la Cátedra para la paz, con la intención de retroalimentar las diferentes concepciones de paz y formas de reconciliación en nuestros territorios. Esta labor puede ser llevada a cabo por el personal directivo y docente, con el propósito de conocer y reconocer experiencias que puedan ser retomadas para fortalecer la convivencia y las relaciones pacíficas en el entorno escolar. Así mismo, recoger las experiencias que cada docente lleva a cabo dentro y fuera del aula para tal propósito.

- Fortalecer el trabajo de las emociones y buscar vías de tramitación, que son convocadas en el aula, como algo natural de los seres humanos, que aparece de manera espontánea. Utilizar videos y cuentos que le permita a los niños, niñas y jóvenes identificar las consecuencias micro y macro de la violencia en la solución de los conflictos personales.

- Incentivar la manifestación de las emociones con respecto a las situaciones que vivencian, desde el acercamiento a sus historias particulares. Si bien el silencio permite no exponerse ante los pares, la catarsis a través de la palabra y la escucha es necesaria para lograr la reconciliación. 
- Generar acciones colectivas a través de proyectos de aula que permitan promover la solidaridad y la compasión por las demás personas que comparten el mismo espacio.

- Enriquecer el trabajo con los dilemas morales que son cotidianos en la vida escolar. Vincular esta estrategia con los contenidos propios de las asignaturas para que los estudiantes comprendan que los contenidos tienen una razón y una conexión con la realidad, que no son conocimientos abstractos.

- Promover, a través de las actividades que se realizan cotidianamente, y entender la relación entre la causa o causas de un conflicto y su consecuencia, sea de tipo económico, político, social, cultural o ideológico.

- Desmantelar estereotipos y prejuicios sobre el otro. Esta labor se puede hacer a través de talleres de sensibilización, encuestas y entrevistas a personas de la comunidad en la cual se encuentra la institución. Permitir que los estudiantes manifiesten sus estereotipos -verdades a medias- para conducir el ejercicio de reflexión que les posibilite llegar a otras comprensiones

- Aportar elementos desde las diferentes asignaturas para que los estudiantes aprendan a leer críticamente la información que se divulga en los medios de comunicación. Así mismo, que puedan ver con otros ojos las producciones audiovisuales, para identificar allí estereotipos que circulan sobre determinados grupos poblacionales.

- Promover espacios de diálogo de saberes, por ejemplo, invitando personas de la comunidad al aula para narrar sus experiencias.

- Invitar a los estudiantes a rastrear experiencias comunitarias de construcción de paz realizadas por grupos juveniles, de mujeres, organizaciones de víctimas y derechos humanos. Incluso, que logren vincularse a ellas.

- Exhortar, a partir de la convicción propia y argumentos sólidos, a que los estudiantes, las familias y otros docentes apoyen la producción de bienes y servicios al interior de la misma comunidad. De esta forma, se fortalecen los lazos y el tejido comunitario. 
Aportes para pensar la Cátedra de la paz desde las resistencias y proyectos de paz de los grupos étnicos en Colombia

- Colocar a decisión colectiva todas las decisiones importantes del grupo. A pesar de que la institución escolar es habitada mayoritariamente por niños, niñas y jóvenes las decisiones son tomadas por adultos. Si bien reconocemos la importancia de la autoridad y la voz experimentada para direccionar el devenir de la institución, es pertinente también que quienes componen el grupo poblacional más grande tengan voz y voto en las decisiones que les afectan directamente. El ejercicio de la anhelada democracia no puede agotarse en un lugar como la escuela, la cual es, sin lugar a duda, el reflejo de la misma sociedad.

\section{UN AMBIENTE DE AULA PACÍFICO A PARTIR DE LAS EXPERIENCIAS DE PAZ DE LOS GRUPOS ÉTNICOS}

Retomando el apartado anterior, consideramos fundamental la democratización del aula. Las relaciones, desde esta perspectiva, deben estar mediadas por condiciones de justicia y respeto; por ello proponemos una estrategia para lograr un ambiente pacífico en el aula retomando los aprendizajes y experiencias de paz de los grupos étnicos. Así, se presentan tres dimensiones para la consecución de este propósito:

- Cada grupo cuenta con un plan de vida: como expusimos anteriormente, las comunidades indígenas formulan sus planes de vida a partir de un proceso participativo de autodiagnóstico; es por lo tanto un pacto social. El plan de vida responde a tres preguntas fundamentales: ¿quiénes somos?, ¿qué buscamos? y ¿cómo lo haremos? ("Los planes de vida", s. f.). En este sentido, proponemos llevar tal propuesta al aula, que resulte en una construcción colectiva y sea el eje que articule las relaciones entre los estudiantes y el cuerpo docente. Para estructurar el plan es necesario llevar a cabo una serie de pasos. En primer lugar, se establece el proceso para elaborar el plan, los procedimientos y las formas en que cada persona participará y tomará decisiones. Se plantea una visión o un propósito que aclare el camino a seguir y, posteriormente, se identifican, describen y priorizan las problemáticas que requieren ser atendidas. Con este diagnóstico elaborado se procede a plantear las estrategias o posibles soluciones para las 
problemáticas o necesidades identificadas, de forma consensuada se delegan funciones y se conforman equipos para cada una de las estrategias a implementar, teniendo en cuenta si estas serán ejecutadas en el corto, mediano o largo plazo. El plan requiere, así mismo, un cronograma de actividades y un proceso de evaluación que puede hacerse durante y después de la implementación; igualmente, de forma participativa. En esta perspectiva, la voz del docente se ubica en un plano de horizontalidad con las voces de los estudiantes.

- Los estudiantes resuelven sus propios conflictos: como parte de la organización del grupo, a partir de la formulación del plan de vida, proponemos la conformación de un consejo o asamblea de estudiantes para tramitar, mediar y resolver los conflictos que se presenten al interior del grupo y con el profesorado. Siguiendo el ejemplo de la Kriss Romaní, este consejo de estudiantes buscará las formas pertinentes para resolver los conflictos, reparar los daños y mantener la armonía y la sana convivencia. Esta estrategia, si se implementa y se acompaña de manera adecuada, puede mediar con el carácter punitivo de las sanciones propias de la escuela, y así mismo prevenir la repetición de tales conflictos. Si bien el docente es quien se encarga de plantear los atributos o cualidades que deben tener quienes conformen este consejo, la elección será realizada por el grupo a través de una votación. En el caso de las instituciones mixtas o coeducativas, este consejo debe estar conformado por hombres y mujeres en proporción con la cantidad de estudiantes del grupo. Consideramos pertinente que la representación se calcule en una persona por cada cuatro estudiantes; o sea, en un grupo de cuarenta estudiantes el consejo estaría conformado por diez estudiantes, además del docente encargado de dicho grupo. Así mismo, si de esas cuarenta personas treinta son hombres y diez mujeres, el consejo debería contar con mínimo tres mujeres.

- El aula es un territorio de paz y alegría: al igual que algunas comunidades afrodescendientes, víctimas de la violencia y el desplazamiento, han denominado sus territorios como "territorios de paz", nuestras aulas pueden ser también territorios de paz. Los conflictos no están relacionados exclusivamente con la violencia; 
Aportes para pensar la Cátedra de la paz desde las resistencias y proyectos de paz de los grupos étnicos en Colombia

estos se presentan en la familia, el trabajo, el grupo de amigos y, por supuesto, en la escuela. En este sentido, se debe promover, al interior de nuestro grupo o grupos de estudiantes, la Cultura de la paz como bien. Esto se manifiesta en el decreto que reglamenta la cátedra en mención, y es necesario hacerlo evidente; por ello proponemos denominar el aula desde esta perspectiva, de forma que quienes la habitan encarnen y vivan día a día la paz, porque están dentro de un territorio que les posibilita establecer relaciones interpersonales sanas y armónicas, aunque no necesariamente libres de conflictos.

\section{REFERENCIAS}

Arenas, H. (2017). Creo en la necesidad de la paz completa. Desde abajo. Recuperado de https://www.desdeabajo.info/colombia/item/31847creo-en-la-necesidad-de-la-paz-completa.html

Arteaga, B. I. (2012). El enfoque diferencial: ¿Una apuesta para la construcción de paz? En Universidad Jorge Tadeo Lozano. Identidades, enfoque diferencial y construcción de paz (pp. 15-40). Bogotá: Fondo para la Sociedad Civil por la Paz, la Democracia y los Derechos Humanos.

Barrios, D., y Hoyos, M. (2008). Sobre los planes de vida de los pueblos indígenas de Antioquia: una perspectiva étnica y zonal para vivir bien. Etnias \& Política, (9), pp. 80-101.

Bolaños, G., y Pancho, A. (2008). Plan de vida: una experiencia de construcción colectiva desde la identidad. En Consejo indígena de Centroamérica et al., Hacia el buen vivir: experiencias de gestión indígena en Centro América, Colombia, Costa Rica, Ecuador y Guatemala (pp. 45-118). Bolivia: Fondo Indígena.

Castillo, L. (2007). Nuevos sujetos étnicos en Colombia. Las comunidades negras y la lucha por sus territorios y su visibilidad en las estadísticas. En M. López, C. Figueroa y B. Rajland, Temas y procesos de la historia reciente de América Latina (pp. 147-170). Buenos Aires: CLACSO.

De Sousa, B. (2006). Conocer desde el sur. Para una cultura política emancipatoria. Lima: Fondo Editorial de la Facultad de Ciencias Sociales - UNMSM.

De Sousa, B. (2009). Pensar el Estado y la sociedad: desafíos actuales. Buenos Aires: Waldhuter. 
De Sousa, B. (2010). Refundación del Estado en América Latina. Perspectivas desde una epistemología del sur. Lima: Instituto Internacional de Derecho y Sociedad.

Escobar, A. (2014). Sentipensar con la tierra. Nuevas lecturas sobre desarrollo, territorio y diferencias. Medellín: Fondo Editorial UNAULA.

Escola de Cultura de Pau (2010). Cuadernos de construcción de paz 12: introducción a los procesos de paz. Barcelona: Escola de Cultura de Pau. Recuperado de http://escolapau.uab.cat/img/qcp/introduccion_procesos_paz.pdf

Estos son los 125 municipios del posconflicto, según la ONU (2015). Recuperado de http://reconciliacioncolombia.com/web/historia/1977/estosson-los-125-municipios-del-posconflicto-segun-la-onu

Galtung, J. (2003). Paz por medios pacíficos. Bilbao: Bakeaz.

Gamboa, J., y Rojas, C. (2005). Notas introductorias sobre las Kriss Romaní o Romaniya: el sistema jurídico transnacional del pueblo Rom. En C. Parra y G. Rodríguez, Comunidades étnicas en Colombia: cultura y jurisprudencia (pags. 181-211). Bogotá: Centro Editorial Universidad del Rosario.

García, D., y Ospina, E. (2016). Orientaciones básicas para la implementación de la cátedra de la paz en las instituciones educativas de Medellín (documento inédito). Medellín: Museo Casa de la Memoria.

Giraldo, O. F. (2014). Utopías en la era de la supervivencia: una interpretación del buen vivir. México: Editorial Ítaca.

Gómez, D. (2010). Pueblo Rrom - gitano- de Colombia. Haciendo camino al andar. Bogotá: Departamento Nacional de Planeación.

Hernández, E. (2000). Comunidades de Paz: expresiones de construcción de paz entre la guerra y la esperanza. Reflexión Política, 2(4), 43-55.

Hernández, E. (2015). Empoderamiento pacifista del actual proceso de paz en Colombia: 2012-2015. Revista de Paz y Conflictos, 8(2), 179-202.

Los planes de vida (s. f.). Recuperado de http://www.territorioindigenaygobernanza.com/planesdevida.html

Muñoz, F., Herrera, J., Molina, B., y Sánchez, S. (2005). Investigación de la paz y los derechos humanos desde Andalucía. Granada: Editorial Universidad de Granada.

Museo Casa de la Memoria (2015). Trazando la paz: un propósito colectivo (documento interno). Medellín: Museo Casa de la Memoria.

Peñas, C. A. (2015). ¿Cómo podemos implementar la "cátedra de la paz" (MEN, 2015) en la educación preescolar? Ruta Maestra, 13, 82-91. 
Aportes para pensar la Cátedra de la paz desde las resistencias y proyectos de paz de los grupos étnicos en Colombia

Propuesta de paz del Movimiento Indígena, una iniciativa para todos (2012). Recuperado de http://www.cric-colombia.org/portal/http://www. cric-colombia.org/portal/

República de Colombia (1993). Ley 99 de 1993. Por la cual se crea el Ministerio del Medio Ambiente. Recuperado de http://www.humboldt. org.co/images/documentos/pdf/Normativo/1993-12-22-ley-99-crea-el-sinay-mma.pdf

República de Colombia (2010). Ley 1424 de 2010. Disposiciones de justicia transicional que garanticen verdad, justicia y reparación a las víctimas de desmovilizados de grupos organizados al margen de la ley. Recuperado de http://www.alcaldiabogota.gov.co/sisjur/normas/Norma1.jsp?i=41055

República de Colombia (2014). Ley 1732 de 2014. Cátedra de la paz. Recuperado de http://wsp.presidencia.gov.co/Normativa/Leyes/Documents/ LEY\%201732\%20DEL\%2001\%20DE\%20SEPTIEMBRE\%20DE\%202014.pdf

República de Colombia (2015). Decreto 1038 de 2015. Cátedra de la paz. Recuperado de http://www.alcaldiabogota.gov.co/sisjur/normas/Norma1.jsp?i=61735

Restrepo, B. (2013). Sobre los diálogos de paz. Documento presentado en el Conversatorio sobre los Diálogos de Paz en La Habana, mayo 29 de 2013. Medellín: Corporación Región. Recuperado de http://www.valenciad. com/files/BRestrepo.pdf

Rosero, M., y Sánchez, J. (2009). Una mirada general al contexto que enmarca los Planes de Vida y Planes de Etnodesarrollo. Recuperado de http://observatorioetnicocecoin.org.co/files/4_Guia_planes_de_vida_y_de_ desarrollo.pdf

Urrego, A. M., y Marín, M. L. (2013). Retos para la educación en un posible contexto de postconflicto político en Colombia. En Instituto Popular de Capacitación, Los retos de la paz y la potencialidad de la memoria para avanzar hacia un proyecto societal democrático e incluyente (pags. 87-112). Medellín: Instituto Popular de Capacitación. 